Application Note

\title{
SimkaMin: fast and resource frugal de novo comparative metagenomics
}

\author{
Gaetan Benoit ${ }^{1}$, Mahendra Mariadassou ${ }^{2}$, Stéphane Robin ${ }^{3}$, Sophie \\ Schbath $^{2}$, Pierre Peterlongo ${ }^{1}$, and Claire Lemaitre ${ }^{1, *}$ \\ ${ }^{1}$ Univ Rennes, Inria, CNRS, IRISA, F-35000 Rennes, France \\ ${ }^{2}$ MalAGE, INRA, Université Paris-Saclay, 78350, Jouy-en-Josas, France \\ ${ }^{3}$ UMR MIA-Paris, AgroParisTech, INRA, Université Paris-Saclay, 75005, Paris, France \\ * To whom correspondence should be addressed. \\ Associate Editor: $X X X X X X X X$ \\ Received on XXXXX; revised on XXXXX; accepted on XXXXX
}

\begin{abstract}
Motivation: De novo comparative metagenomics is one of the most straightforward ways to analyze large sets of metagenomics data. Latest methods use the fraction of shared $k$-mers to estimate genomic similarity between read sets. However, those methods, while extremely efficient, are still limited by computational needs for practical usage outside of large computing facilities.

Results: We present SimkaMin, a quick comparative metagenomics tool with low disk and memory footprints, thanks to an efficient data subsampling scheme used to estimate Bray-Curtis and Jaccard dissimilarities. One billion metagenomic reads can be analyzed in less than 3 minutes, with tiny memory $(1.09 \mathrm{~GB})$ and disk $(\approx 0.3 \mathrm{~GB})$ requirements and without altering the quality of the downstream comparative analyses, making of SimkaMin a tool perfectly tailored for very large-scale metagenomic projects.

Availability: https://github.com/GATB/simka
\end{abstract}

\section{Introduction}

De novo comparative metagenomics is one of the most straightforward ways to analyze large sets of metagenomics data. It consists in estimating pairwise distances between samples, based only on their read content and using no a priori knowledge such as reference databases. In order to scale to very large datasets, all-versus-all sequence comparisons are avoided and the current fastest methods use long $k$-mers as comparison units, with $k>20$. Typically, in the tool Simka (Benoit et al., 2016), each sample is represented as a set of $k$-mers and various distances can be computed based on the amount and abundance of shared and specific $k$-mers between samples. Even if such methods are several orders of magnitude faster than alignment based methods, they are becoming too slow and resource-intensive for today's very-large metagenomic projects, such as the HMP (Lloyd-Price et al., 2017) or TARA Oceans (Bork et al., 2015), therefore limiting their practical use. We present here a new software called SimkaMin that drastically reduces the computational time by subsampling the $k$-mer space. Instead of considering the billions of $k$-mers typically present in metagenomic projects, genomic distances are estimated on randomly picked $k$-mers. $k$-mer subsampling is performed using the Minhash principle (Broder, 1997), that was introduced in the popular genome comparison tool Mash (Ondov et al., 2016). Contrary to Mash, which can only compute presence/absence based distances, SimkaMin extends Minhash to the popular abundance-based Bray-Curtis dissimilarity. Taking $k$-mer abundances into account is indispensable when comparing metagenomics samples with highly variable species diversity profiles or in the general case of raw sequencing datasets where sequencing errors generate many low abundance $k$-mers.

\section{Methods}

Typically, a comparative tool would compute the Bray-Curtis dissimilarity $B C\left(S_{1}, S_{2}\right)$ from the counts of all the $W k$-mers occurring in a given pair of datasets $S_{1}$ and $S_{2}$. Here, the key idea is to compute instead an estimate $\widehat{B C}\left(S_{1}, S_{2}\right)$ of $B C\left(S_{1}, S_{2}\right)$ based on a reduced subset of $w(w \ll W)$ randomly selected $k$-mers and their abundances in each dataset. In order to limit resource use, we never store the $W k$-mers but instead adapt the Minhash (Broder, 1997) principle to select the $w k$-mers on the fly.

Our approach is divided in two steps. First, it computes for each dataset a set of $w k$-mers together with their abundances, hereafter called a sketch Then, it compares sketches in a pairwise fashion. 

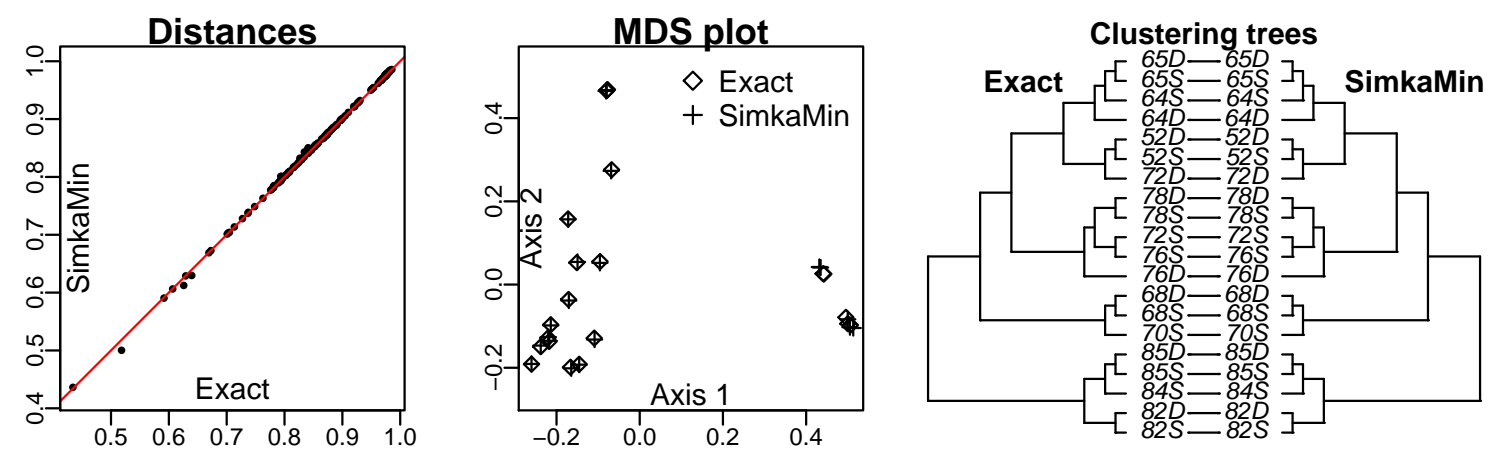

Fig. 1. Comparison of SimkaMin results (with $w=10^{6}$ and default $k$-mer size (21)) with "exact" (i.e. without subsampling) results, in terms of raw distances (left), Multidimensional Scaling projection of the samples (middle) and clustering trees of the samples (right).

Sketching datasets A hash function $h$ is used to transform each $k$-mer of a dataset into a 64 bits integer. These hashes are inserted in a sorted list $L$ of fixed size $w$, that keeps only $w$ distinct $k$-mers, those with the smallest hash values. The resulting $w$ hashes represent a Minhash sketch $m$. Together with the list $L$, we maintain a structure that associates the abundance to each of the $w k$-mers present in $L$.

Distance computation For a pair of datasets $\left(S_{1}, S_{2}\right)$, SimkaMin uses the associated sketches $\left(m_{1}, m_{2}\right)$ to compute the Bray-Curtis dissimilarity $\widehat{B C}\left(S_{1}, S_{2}\right)$. The Bray-Curtis dissimilarity is computed using the counts of the $w$ smallest (in the sense of their hash values) $k$-mers of the union $m_{1} \cup m_{2}$. In practice the two sorted sketches are read in parallel, and the merge stops when $w$ distinct hashes have been seen. Comparing two sketches has a $O(w)$ time complexity since sketches are already sorted.

\section{Accuracy of the approximate $\mathrm{BC}$ dissimilarity}

$\widehat{B C}\left(S_{1}, S_{2}\right)$ is a random estimate of $B C\left(S_{1}, S_{2}\right)$, as it depends on a random selection of $k$-mers. Standard sampling theory shows that both the bias and the standard deviation of $\widehat{B C}\left(S_{1}, S_{2}\right)$ decrease with the sketch size $w$. The bias decreases at speed $1 / w$ and and the standard deviation at speed $1 / \sqrt{w}$ and thus dominates the bias term for large $w$ (see Supp. Mat.).

\section{Usage}

SimkaMin is implemented in $\mathrm{C}++$ and is based on the GATB library (Drezen et al., 2014). The main options are the $k$-mer size and the size $w$ of the sketches SimkaMin can filter out $k$-mers seen only once in dataset, as these $k$-mers are likely to contain sequencing errors. Output files are composed of the Bray-Curtis and the Jaccard dissimilarity matrices. The sketches of all input datasets are stored in a compressed binary file that can be updated later with sketches of additional datasets thanks to a dedicated update python script.

\section{Results}

SimkaMin, was tested on a Tara oceans (Villar et al., 2015) seawater metagenomic set composed of 20 datasets. Reference results are the one without subsampling and were computed using Simka (Benoit et al., 2016). Simka and SimkaMin were both parameterized to limit the computations to the first 50 millions reads of each dataset, thus analysing in total 1 billion reads (see Supp. Mat. for details and command lines)

Results presented in Table 1 show that a tiny amount of resources is sufficient to obtain highly accurate results. With default value $\left(w=10^{6}\right)$, compared to the so-called "exact" approach, SimkaMin provides results $\approx 11$ times faster, with $\approx 3$ times less RAM, and using $\approx 718$ times less disk
Moreover, the bias due to subsampling is predictable and is sufficiently low not to alter the quality of the downstream analyses results, such as multidimensional scaling or sample clustering, as shown in Fig. 1. Similar experiments, performed on other metagenomic datasets from other biomes (soil and human gut), lead to similar conclusions (see Supp. Mat.).

\begin{tabular}{c|cccc}
$w$ value & Time & Mem $(\mathrm{GB})$ & $\begin{array}{c}\text { Disk usage } \\
\text { size (MB) }\end{array}$ & Correlation \\
\hline $10^{4}$ & $2 \mathrm{mn} 49$ & 0.21 & 3 & .9944 \\
$10^{5}$ & $2 \mathrm{mn} 49$ & 0.99 & 31 & .9976 \\
$10^{6}$ & $3 \mathrm{mn} 07$ & 1.09 & 306 & .9991 \\
$10^{7}$ & $6 \mathrm{mn} 59$ & 12.12 & 3,051 & .9994 \\
unlimited & $33 \mathrm{mn} 12$ & 3.71 & $22.10^{4}$ & 1
\end{tabular}

Table 1. SimkaMin performances. $w$ is the number of $k$-mers per sketch. The spearman correlation coefficient is computed between SimkaMin Bray-Curtis dissimilarities versus the non-sampled ones ("unlimited").

\section{Acknowledgment}

This work was supported by the French ANR-14-CE23-0001 Hydrogen Project. Computations have been performed thanks to the resources of the Genouest infrastructure.

\section{References}

Benoit,G., Peterlongo,P., Mariadassou,M., Drezen,E., Schbath,S., Lavenier,D. and Lemaitre,C. (2016) Multiple comparative metagenomics using multiset k-mer counting. PeerJ Computer Science, 2, e94.

Bork,P., Bowler,C., De Vargas,C., Gorsky,G., Karsenti,E. and Wincker,P (2015). Tara oceans studies plankton at planetary scale.

Broder,A.Z. (1997) On the resemblance and containment of documents. In Compression and Complexity of Sequences 1997. Proceedings pp. 21-29 IEEE.

Drezen,E., Rizk,G., Chikhi,R., Deltel,C., Lemaitre,C., Peterlongo,P. and Lavenier,D. (2014) GATB: genome assembly \& analysis tool box. Bioinformatics, 30 (20), 2959-2961.

Lloyd-Price,J., Mahurkar,A., Rahnavard,G., Crabtree,J., Orvis,J. et al. (2017) Strains, functions and dynamics in the expanded human microbiome project. Nature, $\mathbf{5 5 0}$ (7674), 61.

Ondov,B.D., Treangen,T.J., Melsted,P., Mallonee,A.B., Bergman,N.H., Koren,S. and Phillippy,A.M. (2016) Mash: fast genome and metagenome distance estimation using minhash. Genome Biol, 17 (1), 132.

Villar,E., Farrant,G.K., Follows,M., Garczarek,L., Speich,S. et al. (2015) Environmental characteristics of agulhas rings affect interocean plankton transport. Science, 348 (6237), 1261447. 